\title{
An evolutionary habitat selection by the plateau pika (Ochotona curzoniae) in relation to the niche construction of the livestock management on the Qinghai-Tibetan plateau
}

\author{
Migmar Wangdwei ${ }^{1}$, Ma Hong-Mei ${ }^{1}$, La Qiong ${ }^{1}$, Pan Xuan ${ }^{1}$, Tahi Ghaltso ${ }^{1}$, Wu \\ Qianqian $^{1}$, Deching Wangmo ${ }^{1}$, Han Hui ${ }^{1}$, Song Xuanzi ${ }^{1}$, Cao Rui ${ }^{1}$, Liu Mengjun ${ }^{1}$, Meng \\ Huimei $^{1}$, and Jennifer Watts ${ }^{1}$ \\ ${ }^{1}$ Affiliation not available
}

October 22, 2020

\begin{abstract}
Habitat selection by animals is conventionally linked with nature selection pressures. However, niche construction as a potent evolutionary agent palys a crucial role for many types of evolutionary habitat selection by species and this modifies nature selection pressures. We used data obtained from an 11-year period to observe the evolutionary habitat selection by the plateau pika (Ochotona curzoniae) in relation to the niche construction of livestock management. We hypothesized that the niche construction of local nomads affects the evolutionary habitat selection of the plateau pika, and furthermore that the plateau pika does not fit niche conservatism in the Kobresia ecosystem of the Qinghai Tibetan Plateau (QTP). In order to verify the above hypotheses, we used the distance to the nearest yak-bedding areas and dung management as the variables that most strongly explain variation in pika occupancy (probability) and detection probability based on free-ranging livestock. Both pika occupancy and detection probability decrease sharply with increasing distance to the nearest yak-bedding area, suggesting that pika population densities are highest closest to yak-bedding areas. There is a strong correlation between dung cover and occupancy of the plateau pika; the more dung drying on the ground, the more plateau pikas occupy the area. Consequently, the plateau pika is frequently detected in damaged grass. Rangeland dominated by Kobresia sedges on the QTP is modified by livestock grazing and management, especially by dung management. This is the first research suggesting that local nomads may cause expansion of the habitat of the plateau pika. In sum, the habitat of the plateau pika is expanding and there is strong evidence that it retains its biodiversity on the Qinghai-Tibetan Plateau in spite of exposure to poison as control measure. Still, the current control management of the plateau pika may result in diminished diversity of both fauna and flora species and a collapse of the food web on the QTP.
\end{abstract}

\section{Hosted file}

final version 2020.pdf available at https://authorea.com/users/304715/articles/488220-anevolutionary-habitat-selection-by-the-plateau-pika-ochotona-curzoniae-in-relation-tothe-niche-construction-of-the-livestock-management-on-the-qinghai-tibetan-plateau 